\title{
Journeys across the membrane
}

\author{
Nathan Blow
}

\begin{abstract}
From high-throughput electroporation platforms capable of transfecting thousands of different cells in a day, to nanowires that puncture and deliver DNA to just a single cell, new technology is emerging to help researchers with their changing gene delivery needs.
\end{abstract}

The technology used to deliver nucleic acids into cells has undergone a renaissance in recent years. Some scientists think it may have spawned out of a growing interest in studying difficult-to-transfect primary cell types, whereas others suspect the increasing desire of scientists to use high-throughput screening approaches in their research is leading developers to hunt for new ways to deliver DNA and RNA faster and more efficiently. Whatever the reason, novel approaches along with improvements to existing protocols for getting molecules into cells, both in vitro and in vivo, are being reported with greater frequency.

Here we take a look at four recent developments in the field of nonviral nucleic acid delivery technology and explore how these advances are affecting research in areas ranging from stem cell differentiation to drug development.

\section{Finding the right vehicle}

Scientists have developed both physical and chemical methods for nucleic acid delivery. Physical methods, such as electroporation or particle bombardment, involve rapidly changing the cell membrane to allow uptake of DNA or RNA, and chemical approaches often require attachment of the nucleic acids to delivery vehicles, thereby creating complexes that can be shuttled into a cell.

According to Anne-Laure BolcatoBellemin, in vivo research manager at Polyplus-transfection, for chemical approaches the choice of delivery vehicle will often be determined by its cargo. "For us, polymers are more dedicated to gene delivery, while lipids are being used for [small interfering RNA] delivery," she explains.

Polymer-based vehicles, such as polyethylenimine (PEI), polyamine and cyclodextran,

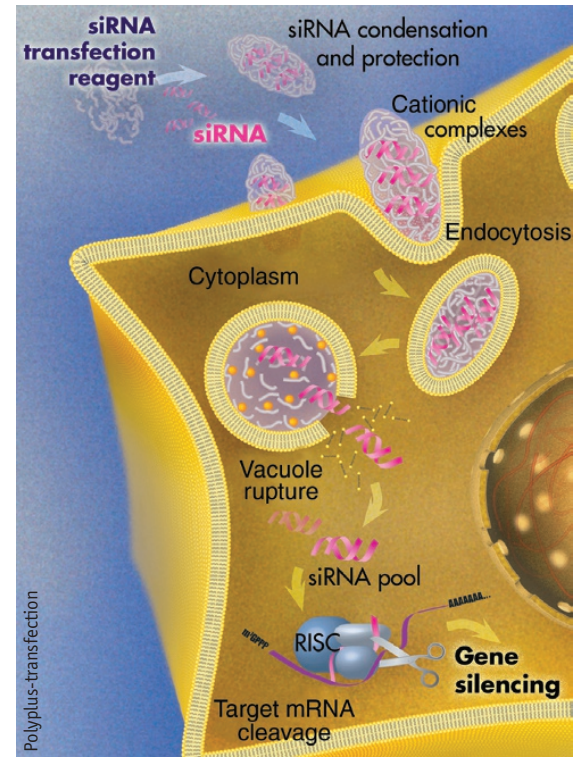

Diagram of the proposed mechanism for cellular uptake of a cationic delivery vehicle carrying siRNA for gene silencing.

act by binding and condensing DNA or RNA into stable particles that can be taken up by cells through endocytosis. Bolcato-Bellemin says that researchers at Polyplus have developed a suite of PEI-based vehicles for in vitro DNA delivery as well as labeled vehicles that can be used to track PEI-DNA complexes within a cell. Other developers_-including Qiagen with their SuperFect and PolyFect transfection reagents, and Fermentas with the ExGen 500 reagent-also provide polymerbased solutions for in vitro cell transfection.

Lipid-based vehicles, which can be conjugated to or surround nucleic acids or other molecules to be taken up by cells, have been used for transfection for more than 20 years and are among the most popular in vitro delivery vehicles. Several well-established lipid-based in vitro DNA transfection reagents, such as InvivoGen's LipoGen and Invitrogen's Lipofectamine 2000 and Optifect-as well as their first-generation Lipofectin and Lipofectamine-are currently available to researchers. But lipids are also finding more and more use as vehicles for the delivery of small interfering RNA (siRNA) into cultured cells. Ambion (now part of Life Technologies Corporation) has developed a lipid-based siRNA delivery reagent called siPORT NeoFX, Invitrogen has modified their cationic lipid formulation in Lipofectamine for siRNA, Mirus Bio has developed a combination cationic lipid and polymer-based siRNA transfection reagent called TransIT-TKO and Polyplus-transfection has made available the INTERFERin siRNA transfection reagent.

Transfection of cultured cells is not the only area for nucleic acid vehicle development as some scientists have turned their attention from cultured cells to in vivo delivery. Several biotechnology and pharmaceutical companies, including Polyplus-transfection, Altogen Biosystems, Alnylam Pharmaceuticals and Calando Pharmaceuticals, are now using cationic lipids, biodegradable polymers and liposomes as vehicles to guide pieces of DNA or siRNA around the human body for delivery to specific cell types. Several of these vehicles are now undergoing clinical trials and new vehicle development continues to be a rapidly growing field, with new carrier molecules on the horizon. Robert Langer's group at the Massachusetts Institute of Technology recently reported the construction of a combinatorial library of lipid-like materials that they call lipidoids to be potentially used in siRNA delivery ${ }^{1}$. Still, both polymer and lipid vehicles have drawbacks when it comes to in vivo delivery. Whereas lipids are efficient 

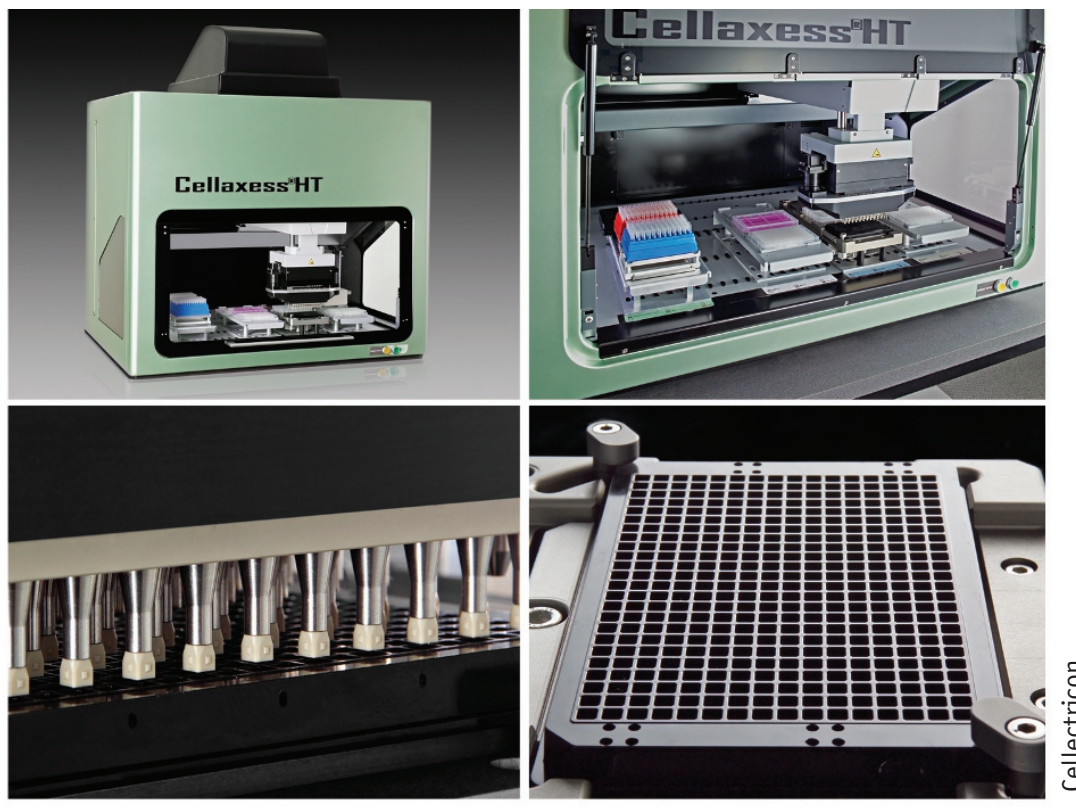

The Cellaxess HT system was designed for high-throughput transfection to enable more effective RNAi screening.

delivery vehicles with effects that tend to last longer than polymers, according to BolcatoBellemin, lipids also tend to induce more immune response than the polymers, which is problematic for therapeutic purposes.

These drawbacks have led to the emergence of nanoparticle vehicles in recent years as an alternative to potentially circumvent some delivery issues. But at the outset, the biggest benefits of using nanoparticle vehicles for in vivo gene delivery might be in what researchers can see by using these little particles. It turns out that the iron oxide core within some newly developed nanoparticles can be used to image DNA or siRNA delivery within the body using magnetic resonance imaging technology, giving researchers a much clearer understanding of delivery efficiency and targeting.

Some developers have entered the world of nanoparticle-based gene delivery. In 2007, Sigma Aldrich introduced the N-TER Nanoparticle siRNA Transfection system, which allows for the conjugation of an siRNA to the surface of the nanoparticle through the N-TER peptide for in vitro delivery applications, and two other developers, $\mathrm{OZ}$ Biosciences and nanoTherics, are now offering magnetic nanoparticles for the delivery of nucleic acids to cells.

In the end, regardless of the vehicle, Bolcato-Bellemin says that when it comes to nonviral delivery of nucleic acids, the goal is straightforward. "The most important thing is to transfect the highest percentage of cells with the lowest amount of DNA or RNA possible [while still achieving the desired result]," she says, adding that in this way problems such as cell toxicity, off-target effects and immune response can be greatly reduced or even eliminated altogether.

\section{Close contact}

"I think of [our approach] in many ways as mimicking what nature does," says Lonnie Shea, professor in the chemical and biological engineering department at Northwestern University, when discussing his group's work on substrate-mediated gene delivery.

The idea behind Shea's delivery method is simple: if a DNA-delivery vehicle complex is tethered to a substrate surface to which the cells are adhering, the cells and delivery vector are effectively co-localized, which should result in more efficient gene delivery. But his major challenge over the past few years has been to fine-tune the associations between the delivery complex and substrate.

Shea's group initially opted for a biotinavidin interaction to tether polymer complexes of DNA to the substrate surface but finding optimal combinations took time. On one hand, he says: "If you have too much biotin on the complex, then the binding is too strong, and the cells cannot internalize the DNA." On the other hand, the use of too little biotin results in mass mobilization, and decreases efficiency and localized delivery.

Shea has also investigated the immobilization by nonspecific interactions between the transfection reagent and the surface, and their data suggest that effective gene delivery results from displacement of the complexes from the substrate by adsorbing proteins. "If we immobilize complexes and wash with phosphate-buffered saline the complexes are stable and do not come off the surface. But if you add serum proteins, then you see the complexes being displaced to the surface."

As displacement of complexes appears to be necessary for uptake by cells, Shea says it is important to have proper substrate surface composition. "If the associations are too strong, then uptake is limited and the complexes may lose their architecture, which limits their efficacy," he says.

Shea has transfected DNA in polymer complexes, lipid complexes, as well as in lentiviruses attached to the substrate surface. One of the benefits of such close contact between the cells and delivery vehicle is that less DNA is needed for transfection, enhancing delivery efficiency.

Shea's group is now moving toward in vivo applications using his substrates. "What the substrate will do is retain the DNA for longer periods of time, preventing it from being degraded," he says, which could improve gene delivery in clinical applications in which complexes and viral vehicles are often rapidly cleared from the body. "An advantage of this approach is that the material can be processed by a variety of techniques, and then the vectors can be immobilized, which stabilizes and increases the activity of the vector. The material can then be implanted, and we can look at the effects when those complexes are delivered locally and stabilized against nuclease activity."

But as others have discovered with siRNA delivery, delivery in the in vivo world is very different from in vitro. "We have had great success in vitro with efficient delivery to a variety of cell types, but in vivo the environment around the cell is different, creating new challenges," notes Shea.

\section{Getting more physical}

For Johan Pihl, product manager at Cellectricon, it took the advancement of technology in another field to help him see the next step for his company. "We believed that we had this very potent method for gene delivery, but at the time we did not know quite what to do with it," says Pihl.

That was five years ago. Then Pihl and his colleagues started to see the RNA interference (RNAi) field "cook" with more and more researchers interested in doing high-through- 


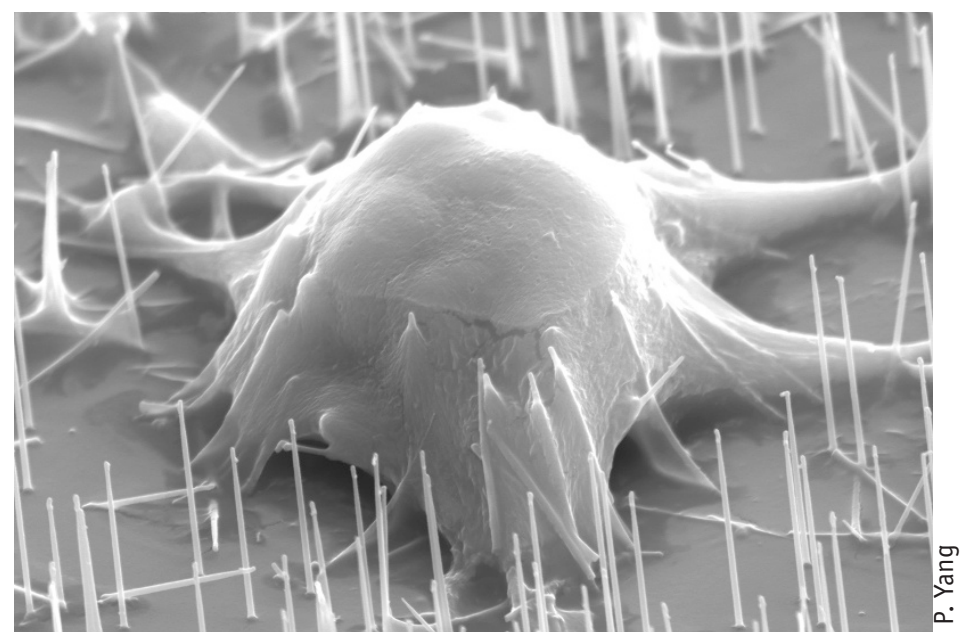

Gene delivery can be accomplished by culturing cells on a bed of nanowires that have DNA on the surface.

put screens along with core screening centers springing up around the world, and he knew their next step. "We realized this was where a high-throughput transfection approach was most needed since transfection is one of the major bottlenecks in RNAi screens."

So in 2006 developers at Cellectricon started working on plans to adapt their Cellaxess gene delivery technology — which is based on electroporation where electric fields permeabilize cell membranes to deliver DNA or RNA-into a high-throughput automated instrument.

"We use a capillary tube equipped with electrodes to deliver electric fields to the cells," explains Pihl. The idea for their new instrument was to take advantage of the small size of these capillary tubes by bringing a series of them into close proximity to adherent cells located in individual wells on a microtiter plate. An electrical field could then be applied and delivered to each individual capillary to transfect multiple cell populations.

Over the next two years engineers at Cellectricon added the necessary automation components to their instrument. These additions allowed for the transfer of buffers, addition of siRNA to the cells before transfection, switching of liquid-handling tips with electroporation units to electroporate cells and finally the addition of cell culture media for recovery after transfection. The system, called Cellaxess HT, was introduced in April 2008 and is capable of transfecting 50,000 wells of cells per day.

Other companies have also developed high-throughput solutions for gene delivery. The bioscience division of Lonza now provides the 96 -well microtiter plate
Nucleofector kit for transfection of stem cells, which like the Cellaxess HT system uses electroporation. Another company, MaxCyte, has developed an electroporationbased approach in which cells in a flowing stream are passed through an electroporation unit. Both the capillary electrode approach taken by Cellectricon and the flow design of MaxCyte enable lower currents to be used than traditional electroporation approaches, resulting in the generation of less heat and improved cell viability after transfection.

High-throughput transfection systems could make RNAi screening faster and more efficient in the future, but it is only one part of the process. "Once you overcome the transfection bottleneck, you still have the problem of establishing a stable and good assay that will give you hits at the end of the day," says Pihl. But he hopes that having instruments dedicated to transfection will give researchers more time to focus on their downstream assays.

\section{Sticking it to them}

Peidong Yang, professor of chemistry at the University of California, Berkeley, thought his new delivery approach was a long shot. "My intuition was that it would kill the cell," he recalls. And who could blame him? The idea of puncturing a cell with an electrical wire to deliver DNA does seem a bit extreme at first.

The idea actually came from an informal chat between Yang and his colleague Bruce Conklin, a professor also at University of California, San Francisco, a couple years ago. Conklin was interested in using electrodes 
to look at stem cell differentiation, while Yang's group had been working on nanowire-based nanoelectronics and probes. "That was the starting point: nanowires as electrodes for guiding stem cell differentiation," says Yang.

But first things first, and for Yang and his group this meant determining whether stem cells could be punctured with nanowires and actually survive. "What we saw was that with sub-80-nanometer nanowires the cells were ok," says Yang. "At least in terms of not changing the lifetime of the stem cell significantly." But there was a strong size dependency in the cell's ability to survive puncture with the nanowire; wires over 200 nanometers killed cells.

Armed with the knowledge that cells can in fact survive with unaltered lifetimes, Yang fabricated an array of 80 -nanometer nanowires functionalized with DNA to test whether the wires could also deliver DNA. Although the cells survived the puncture and could take up DNA, the results showed that these nanowires may not be ready for most researchers' gene delivery needs yet. "I mean we are talking about a low percent of transfected cells. It is very inefficient," notes Yang. He was not surprised by the low efficiency because the nanowires were made of silicon and the DNA was physically absorbed to the surface-not the most efficient possible attachment. Still, for his and Conklin's purposes the efficiency was more than adequate, giving them the ability not only to deliver small molecules on the nanowires but also electrically stimulate the stem cells at the same time.

"Electrical stimulation of stem cells is another way to think about how to program the cell differentiation," says Yang. Although it is still very early in their research, and not much is known about the effects of electrical stimulation on stem cells, Yang is encouraged by the fact that they now have a single platform to test both chemical and electric stimulation.
And for those researchers and developers interested in improving upon the current efficiency of nanowires for gene delivery, Yang thinks changing the surface of the wire could be the way to go. "We are suggesting that if anyone wants to improve the efficiency, one can think about using surface functionalization to covalently attach DNA and maybe use photochemistry to cleave those bonds inside the cell."

While polymer, lipid and nanoparticle delivery reagents along with electroporation techniques continue to be refined and improved, the newer approaches, such as nanowires and substrate mediated delivery, demonstrate that there still is a lot to learn when it comes to gene delivery.

1. Akinc, A. et al. A combinatorial library of lipid-like materials for delivery of RNAi therapeutics. Nat Biotechnol. 26, 561-569 (2008).

Nathan Blow is the technology editor for Nature and Nature Methods (n.blow@boston.nature.com). 
TECHNOLOGY FEATURE

SUPPLIERS GUIDE: COMPANIES OFFERING GENE DELIVERY

PRODUCTS AND SOLUTIONS

\section{Company}

Ambion Inc

Altogen Biosystems

Applied Biosystems

Asuragen

BD Biosciences

Bio-Rad Laboratories Cenix

BioSciences

CombiMatrix

Dharmacon

Eurogentec

Exiqon

GeneTools

genoway

Imgenex

Integrated DNA Technologies

Invitrogen

Invivogen

Cellectricon

Lonza/Amaxa biosystems

MaxCyte

Mirus Bio

MWG Biotech

NanoLab Inc

nanoTherics

New England Biolabs

Ocean Nanotech

OligoEngine

Thermo Fisher Open Biosystems

OZ Biosciences

Tecan Group LTD

Polyplus-transfections

Promega

Qiagen

Sigma Aldrich

Stratagene

Sylentis

Targeting Systems

Vectalys
Web address

http://www.ambion.com/

http://www.altogen.com/

http://www.appliedbiosystems.com/

http://www.asuragen.com

http://www.bdbiosciences.com/

http://www.cenix-bioscience.com/

http://www.combimatrix.com

http://www.dharmacon.com/

http://www.eurogentec.com/

http://www.exiqon.com/

http://www.gene-tools.com

http://www.genoway.com/

http://www.imgenex.com/

http://www.idtdna.com/

http://www.invitrogen.com/

http://www.invivogen.com/

http://www.cellectricon.com/

http://www.amaxa.com/

http://www.maxcyte.com/

http://www.mirusbio.com/

http://www.mwg-biotech.com/

http://www.nano-lab.com/

http://www.nanotherics.com/

http://www.neb.com/

http://www.oceannanotech.com/

http://www.oligoengine.com/

http://www.openbiosystems.com/

http://www.ozbiosciences.com/

http://www.tecan.com/

http://www.polyplus-transfection.com/

http://www.promega.com/

http://www1.qiagen.com/

http://www.sigmaaldrich.com/

http://www.stratagene.com/

http://www.sylentis.com/

http://www.targetingsystems.com/

http://www.vectalys.com/ 